ppi $201502 Z U 4645$

Esta publicación científica en formato digital es continuidad de la revista impresa ISSN-Versión Impresa 0798-1406 / ISSN-Versión on line 2542-3185Depósito legal pp $197402 Z$ U34
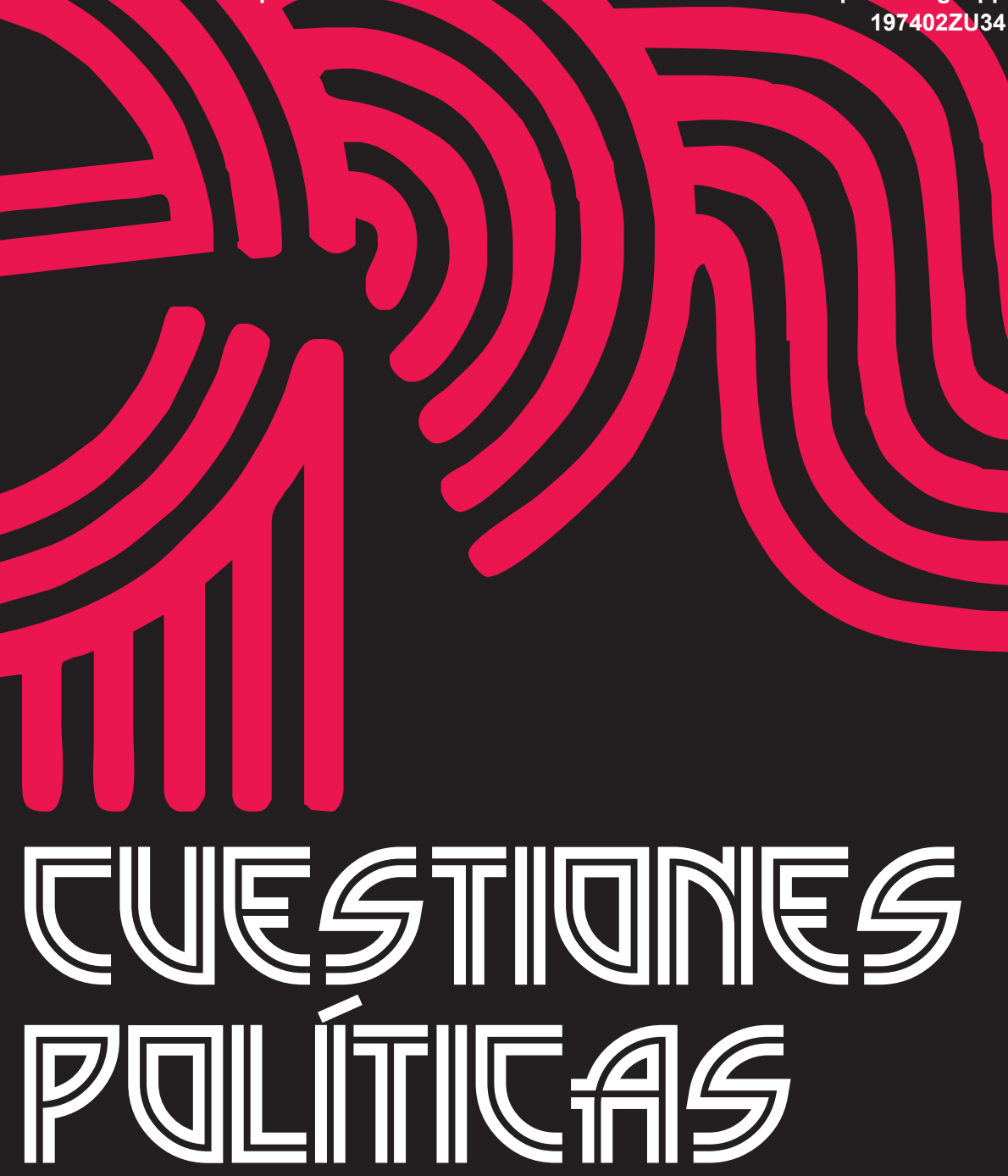

Instituto de Estudios Políticos y Derecho Público "Dr. Humberto J. La Roche" de la Facultad de Ciencias Jurídicas y Políticas de la Universidad del Zulia Maracaibo, Venezuela
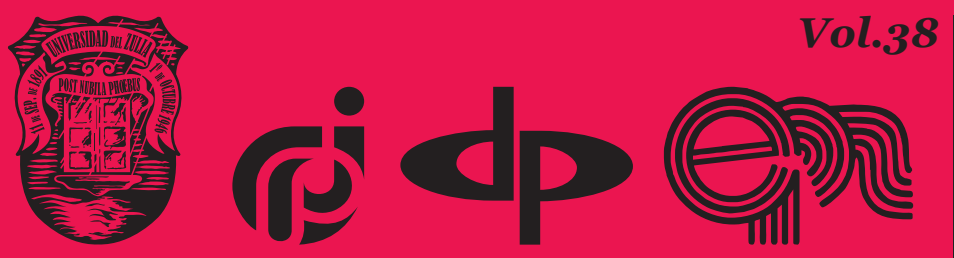

$N^{\circ}$ Especial 2da Parte 2020 


\title{
Influencia de la innovación en la competitividad de las medianas empresas del Ecuador
}

\author{
DOI: https://doi.org/10.46398/cuestpol.382e.36
}

\author{
Marcia Fabiola Jaramillo Paredes * \\ Oscar Mauricio Romero Hidalgo ** \\ Martha Cecilia Aguirre Benalcázar *** \\ Jonathan Alexander Ruiz Carrillo ****
}

\section{Resumen}

Este estudio tuvo como objetivo analizar la influencia de la innovación en la competitividad de las medianas empresas del Ecuador. Los autores consideran a la innovación como la acción de generar ideas a través de la gestión del conocimiento mediante el aprovechamiento de la tecnología, con la finalidad de incidir en la competitividad de las empresas mediante la generación de valor agregado en la oferta de productos y servicios. La metodología del presente artículo se desarrolló como un diseño no experimental de tipo transversal, exploratorio y fundamentalmente bibliográfico para referenciar diferentes textos y contextos empresariales. En esencia, se trató de un trabajo de revisión teórica próximo a la hermenéutica. Entra las conclusiones más representativas se destaca que, la innovación como proceso integral que busca desarrollar al máximo las capacidades productivas de una empresa determinada tiene variadas implicaciones económicas y de competitividad, porque, por un lado, aumenta la cantidad y calidad de los bienes y servicios que se producen al mismo tiempo reduce los costos; por el otro, incide en el abanico de opciones que los pymes otorgan a sus clientes para satisfacer sus necesidades y deseos con ofertas de calidad en un contexto de creciente competencia nacional y global.

* Magíster en Administración de Empresas - UTMACH, Universidad Técnica de Machala. Profesora Titular de la Universidad Técnica de Machala - Ecuador. ORCID ID: https://orcid.org/oooo-00020477-042X. Email: mjaramillo@utmachala.edu.ec

** Doctor en Ciencias Administrativas - UNMSM. Magíster en Dirección de Empresas - UASB. Profesor Titular de la Universidad Técnica de Machala - Ecuador. ORCID ID: https://orcid.org/oooo-0oo3o018-5546. Email: oromero@utmachala.edu.ec

*** Doctora en Ciencias Administrativas - UNMSM. Máster en Administración de Empresas - UTMACH. Profesora Titular de la Universidad Técnica de Machala - Ecuador. ORCID ID: https://orcid.org/ooooo003-4210-795X. Email: maguirre@utmachala.edu.ec

****Doctor en Ingeniería Industrial - UNMSM. Magíster en Docencia Universitaria e Investigación Educativa - UTMACH. Director de la Unidad de Investigación Desarrollo e Innovación Tecnológica del Instituto Superior Tecnológico El Oro - Machala - Ecuador. ORCID ID: https://orcid.org/oooo-00029183-0o04. Email: jruiz@utmachala.edu.ec 
Marcia Jaramillo Paredes, Oscar Mauricio Romero Hidalgo, Martha Cecilia Aguirre Benalcázar y Jonathan Alexander Ruiz Carrillo

Palabras clave: innovación y competitividad; medianas empresas en Ecuador; pymes; política de estimula a la innovación; gerencia del conocimiento.

\title{
Influence of innovation on the competitiveness of Ecuador's medium-sized enterprises
}

\begin{abstract}
This study had as an analysis of the influence of innovation on the competitiveness of medium-sized enterprises in Ecuador. The authors to innovation as the action of generating ideas through the management of knowledge of the use of technology, in order to influence the competitiveness of companies through the generation of added value in the offer of products and services. The methodology of the present article was developed as a non-experimental design of a transversal, exploratory and fundamentally bibliographic type to reference different texts and business contexts. In essence, it was a review work close to hermeneutics. The most reserve conclusions include that innovation as a comprehensive process that, seeking to make the most of the productive capacities of a given company, has implications and competitiveness, on the one hand, increases the quantity and quality of the services and goods that occur at the same time reducing costs; on the other hand, it affects the range that SMEs give their customers for their needs and desires with quality offers in a context of increasing national and global competence.
\end{abstract}

Keywords: innovation and competitiveness; medium-sized enterprises in Ecuador; SMEs; innovation-boosting policy; knowledge management.

\section{Introducción}

La competitividad empresarial es el resultado de la interacción de factores internos y externos de las empresas (Otero y Taddei, 2018) conlleva a que las empresas se mantengan a la vanguardia del mercado; beneficia a las organizaciones en el sentido de incrementar ingresos económicos, además de que brinda una la oportunidad de cambiar o mejorar el estándar de vida las personas a través de la innovación (Ibarra et al., 2017).

En las empresas, los factores exógenos pueden afectar el crecimiento de los niveles de competitividad, sin embargo el sostenimiento de la 
empresa depende mucho de su administración (Mora et al., 2015) y ante un mercado cambiante la planificación estratégica es una herramienta que beneficia en el sentido de que la identificación oportunidades propenda a la implementación de cambios en las organizaciones a fin de cumplir objetivos mediante estrategias ejecutadas que propendan a la sostenibilidad de las unidades económicas (García et al., 2017).

El mundo globalizado implica un gran desafío para todo tipo de empresas, porque existe un mercado cada vez más agresivo en cuanto a la competencia, en donde las entidades de menor tamaño se enfrentan retos de mayor incidencia, es por ello que para lograr mantenerse activas y competitivas es importante la adopción de factores como la implementación de la innovación, para generar procesos de automatización que estimulen la productividad, además de efectuar asignación presupuestaria para la inversión tecnológica, fortalecimiento de las competencias del capital humano y el adecuado uso de los recursos financieros (Molina y Sánchez, 2016).

Es importante acotar, además, que la inversión en tecnología reduce costes de producción y favorece a la calidad de los productos conjuntamente de que genera ventaja competitiva (Ospina et al., 2014). Por lo demás, aun cuando la literatura refiera los beneficios que otorga la adopción tecnológica, no todas las empresas logran automatizar procesos, este panorama se reflejan en las pequeñas empresas, entes que desde sus inicios parten de procesos frágiles de constitución y tienen recursos muy limitados para invertir en el desarrollo de sus capacidades productivas.

A nivel de Latinoamérica, la estructura empresarial en su mayor parte se constituye por las pequeñas y medianas empresas (pymes), realidad de la que también es parte Ecuador, país en donde las empresas denotan inadecuadas formas gestión empresarial, lo cual salta a la vista al evaluar la estructura organizacional pues es muy frecuente que la administración se lleve sin el establecimiento de organigramas y, en algunos casos, sean carentes de la aplicación de un manual de funciones (Tobar, 2015). Sin embargo, a pesar de sus debilidades intrínsecas, algunas empresas de la provincia del Guayas buscan mantener su competitividad a través de calidad de los servicios y productos que generan, así como mediante las negociaciones en mercados aliados, pero, "su talón de Aquiles" se visualiza en los costos elevados (Carvache et al., 2018).

Frente a estas circunstancias, como se menciona en líneas anteriores es necesario que las pymes desarrollen planes estratégicos, para que exploten en mayor grado la oferta de su producción mediante la diversificación e innovación para incrementar su nivel de competitividad dentro del mercado globalizado, pero el sentido de tener presencia como país competitivo implica un trabajo asociativo entre sectores empresariales, entidades del gobierno nacional así como la población en general para que sumen 
Marcia Jaramillo Paredes, Oscar Mauricio Romero Hidalgo, Martha Cecilia Aguirre Benalcázar y Jonathan Alexander Ruiz Carrillo

esfuerzos a fin de impulsar el desarrollo empresarial y económico de la nación (Cadena et al., 2018). Mucho más cuando el desarrollo de las fuerzas productivas de las pequeñas y medianas empresas es un objetivo político que beneficia a la sociedad en su conjunto.

A nivel interno la competitividad no solo depende de los directivos de la empresa, sino que también implica a todo el recurso humano que labora dentro de la empresa, pero también obedece a las relaciones que se mantengan con agentes externos, tales: clientes y autoridades (Gutiérrez y Almanza, 2016). Es por ello, que cada entidad debe de mantener intacta la filosofía que la competencia es el motor empresarial que brinda a toda compañía la oportunidad de trabajar desde una perspectiva destacable en un mercado flexible, lo que propicia a la innovación como un fenómeno concomitante (Barrios et al., 2019).

El alcanzar adecuados estándares de competitividad, demanda de prácticas que favorezcan la introducción de mejoras continuas en todas las dimensiones de la realidad empresarial, que van desde la gerencia y administración, hasta la forma como los empleados se relacionan en la faena productiva (Gil , 2017) porque en el mercado cada ente compite para tener presencia en el entorno y sacar el mayor provecho con el menor costo (Horta et al., 2015), por lo cual, reluce la pregunta de investigación: ¿Qué relación conecta a la innovación y la competitividad en las empresas ecuatorianas? Interrogante que se plantea con la finalidad de determinar la influencia de la innovación frente a la competitividad en las medianas empresas, en un país que en el 2016 las empresas de este tamaño abarcaron el 76,59\% (INEC, 2016).

\section{Materiales y Métodos}

En el contexto del tema tratado en el presente artículo se han revisado bases referenciales sobre la innovación como factor que estimula la competitividad. Esta investigación se desarrolló desde un diseño no experimental de tipo transversal, exploratorio y fundamentalmente bibliográfico para referenciar diferentes textos y contextos. Conviene destacar que el criterio para seleccionar las fuentes documentales, en la modalidad de artículos científico o monografías sobre el tema, estuvo estrechamente relacionada a las variables e indicadores que surgen del objetivo de investigación, tal como se muestra en la siguiente tabla: 
Tabla No. 1.

Resumen de los indicadores considerados por cada variable.

\begin{tabular}{|c|c|}
\hline Variables & Indicadores \\
\hline \multirow{9}{*}{ Innovación } & Gestión del Conocimiento \\
\hline & Factor interno \\
\hline & Factor externo \\
\hline & Calidad \\
\hline & Tecnología \\
\hline & Procesos \\
\hline & Recursos \\
\hline & Clientes \\
\hline & Rentabilidad \\
\hline \multirow{8}{*}{ Competitividad } & Participación empresarial \\
\hline & Mercado local \\
\hline & Factores productivos \\
\hline & Recursos \\
\hline & Valor agregado \\
\hline & Innovación \\
\hline & Estructura productiva \\
\hline & Oferta innovadora \\
\hline
\end{tabular}

Fuente: Elaboración propia. Datos alcanzados en el estudio.

Tal como señala Arias (2006), el diseño documental de investigación se sustenta en un conjunto de operaciones contentivas al: análisis, interpretación, contrastación y crítica de fuentes documentales de diversa naturaleza, con la finalidad de aportar nuevos o renovados saberes sobre la síntesis y organización que surge de una aproximación teórica, lo que hasta cierto punto posiciona nuestra investigación en los dominios de la hermenéutica, que sitúa a los textos en sus contextos de enunciación con condición de posibilidad para desarrollar un tema o problema de estudio. 
Marcia Jaramillo Paredes, Oscar Mauricio Romero Hidalgo, Martha Cecilia Aguirre Benalcázar y Jonathan Alexander Ruiz Carrillo

\section{Influencia de la innovación en la competitividad de las medianas empresas del Ecuador}

La competitividad, es el resultado dela combinación devarios factores que involucra la capacidad del gerente en el sentido de administrar los recursos de una empresa (Rubio y Buitrago, 2019). Además, implica la capacidad de crear nuevos productos a menor costo y en menor tiempo (Vázquez et al., 2014), es por ello por lo que se: "Requiere un equipo directivo dinámico, actualizado, abierto al cambio organizativo y tecnológico, y consciente de la necesidad de considerar a los miembros de la organización como un recurso de primer orden al que hay que cuidar" (Leyva et al., 2018: 17).

Toda la evidencia empírica y teórica demuestra que una empresa crea ventaja competitiva cuando se inserta en un proceso sostenidito de productividad y dota de niveles sufrientes de calidad a los bienes y servicios que produce y reproduce, por ende, su evaluación se efectúa bajo estrictos estándares (López, 2016) y se mide en función de aspectos económicos, humanos y físicos (García el at., 2017). Es por ello, que las organizaciones empresariales, en todos sus niveles y modalidades de existencia, deben de prestar especial atención al mejoramiento de las actividades del quehacer empresarial, lo cual se alcanza mediante objetivos estratégicos bien definidos, esto es, definidos al calor de las aspiraciones y necesidades de sus clientes.

Bajo esta concepción proactiva, reluce el hecho que en su interior las empresas tienen la apremiante tarea de armar las condiciones idóneas de actuación en el rango de su direccionamiento empresarial, teniendo sobre todo- muy en claro que la competitividad no sólo hace referencia al producto, sino también, involucra a distintos procesos objetivos y subjetivos, que empiezan desde el interior de las organizaciones.

En este sentido, es importante tener presente la relevancia de la inteligencia empresarial, misma que se materializa a través de la gestión del conocimiento, porque conlleva al fortalecimiento de las habilidades del talento humano a través de la capacitación de la fuerza de trabajo, de tal manera que permita que este recurso intangible propicie al aprovechamiento de la innovación tecnológica como fuente de estimulación hacia la ventaja competitiva y de productividad (Didriksson, 2015; Fuentes et al., 2016)

Lo anterior, deja entrever que el conocimiento representa un activo importante en las empresas, que, fusionado con la implementación de las tecnologías de información y comunicación en sus procesos productivos, canaliza a la innovación y se torna en tanto elemento de la inteligencia empresarial como eslabón de la competencia y es catalogada como parte de la gestión del conocimiento. 
Por su parte, el término de calidad también está relacionado con la competitividad, pues en torno a este giran el conjunto de acciones, planes, decisiones estratégicas en las empresas. Hoy en día la apremiante globalización permite que las negociones traspasen fronteras, empero las dinámicas de las transacciones se efectúan bajo diferentes estándares siendo uno de ellos las normas International Organization for Standardization (ISO) con la finalidad de estandarizar procesos que verifiquen que el producto está cumpliendo con las necesidades de los clientes, los cuales son cada vez más exigentes y el estatuto de certificación de cierta forma permite a las empresas competir abiertamente (Botello, 2016).

Ahora bien, en el nivel externo de las empresas factores exógenos inciden de manera directa en las organizaciones y, por ende, propician a influenciar en lo referido a la competencia, pues aspectos como las normas jurídicas, situación económica del país, condiciones del mercado (MoraRiapira et al., 2015; Durán, et al.,2018) se muestran como externalidad para las organizaciones empresariales las cuales pueden resultar de cierto modo no tan favorables.

Además, el hecho de competir en el mercado implica conocer a quienes se encuentran en el mismo rol de actuación, precisamente en este punto la tecnología juega a favor, pues por medio de las redes sociales las empresas pueden obtener información sustanciosa que refiera sobre los movimientos de sus competidores (Cepeda y Velásquez, 2017) y, con aquel insumo de datos, plantear estrategias de actuación. En este sentido, es menester señalar que siempre las organizaciones deben de aplicar su respectivo diagnostico situacional porque les reconoce fortalezas mediante la captación de oportunidades que derivan en resultados positivos en distintos roles de actuación, en donde las finanzas se pueden mostrar con ventaja frente a otras empresas lo cual se vea reflejado mediante las utilidades generadas tornando aquello una ventaja competitiva (Tarapuez et al., 2019).

\section{Conclusiones y recomendaciones}

Las fuentes revisadas demuestran claramente que, la innovación como fenómeno integral que busca desarrollar al máximo las capacidades productivas de una empresa determinada tiene variadas implicaciones económicas y de competitividad, ya que, por un lado, aumenta la cantidad y calidad de los bienes y servicios que se producen y, al mismo tiempo, reduce los costos al hacer más eficiente y eficaz el proceso productivo. Por el otro, la innovación incrementa el abanico de opciones que las pymes otorgan a sus clientes para satisfacer sus necesidades y deseos con ofertas de calidad en un contexto de creciente competencia, nacional y global. 
Marcia Jaramillo Paredes, Oscar Mauricio Romero Hidalgo, Martha Cecilia Aguirre Benalcázar y Jonathan Alexander Ruiz Carrillo

Por todo que lo que representa para la economía empresarial y para las finanzas públicas, en particular, la innovación es también un asunto político de primer orden, de hecho, en Ecuador según datos aportados por la Secretaria de Educación Superior, Ciencia, Tecnología e Innovación SENESCYT (2019), el gobierno ecuatoriano promueve el llamado acuerdo 2030 como un espacio de concertación entre el sector industrial, la academia y las distintas instancias de gobierno con alguna competencia en la materia para construir un país más competitivo. Además, la política de innovación ecuatoriana está en completa sintonía con los propósitos de Nacionales Unidades ya que el acuerdo:

(...) está articulado con los Objetivos de Desarrollo Sostenible que impulsa la Organización de Naciones Unidas, se concretará con base en tres acuerdos: una estrategia de innovación abierta que vincule a los actores del sistema de innovación, ciencia y tecnología; la construcción de un fondo permanente de investigación, desarrollo tecnológico e innovación; y, el compromiso de avanzar en la transformación digital (SENESCYT, 2019: s/p.).

La anterior cita pone en evidencia que la innovación se constituye en un fenómeno multidimensional que no debe reducirse a sus implicaciones empresariales, sino que demanda por parte de los investigadores acuciosos, de la estructuración de un modelo teórico y metodológico amplio que apueste además por el diálogo interdisciplinario para dar cuenta de la innovación en sus facetas: técnico-científica, económica-empresarial y como política de estado que busca introducir mejoras continuas en la pymes y la sociedad en su conjunto al calor, de los desafíos que imponen los mercados.

Efectivamente, los cambios introducidos en el orden internacional por la pandemia de COVID-19 pueden desembocar incluso en la introducción de nuevo modelos económicos y políticos inciertos tal como señalan (ArbelaezCampillo et al., 2019; Villasmil, 2020), ante esta situación una de las estrategias empresariales más adecuadas es la innovación como condición de posibilidad para no perecer ante las transformaciones estructurales de los mercados nacionales e internacionales. Y es que la competitividad de las empresas demanda que se apliquen mejoraras continuas en todas las dimensiones del proceso productivo, en un contexto en el cual, las pymes que no están a la vanguardia de las demandas del mercado y no logran potenciar sus capacidades ven reducidas sus oportunidades de negocios o incluso se van a la quiebre.

En conocimiento de estas y otras realidades que viene a determinar el funcionamiento de las pequeñas y medianas empresas en Ecuador, se efectúan las recomendaciones siguientes:

- Potenciar los vínculos que se dan en el triángulo innovación, estructura empresarial y políticas públicas para el logro del desarrollo sostenible. 
- Hacer de la innovación una cultura empresarial que se oponga a las gerencias estáticas y tradiciones que, temen impulsar mejoras sustanciales en los procesos productivos.

- Fomentar el desarrollo de investigaciones en clave interdisciplinaria desde un concepto holístico de innovación que busca expandir su alcance y significado a todas las actividades humanas.

En este orden de ideas, no debe suponerse tampoco que la innovación es simplemente una moda o una tendencia teórica que no responde a las necesidades del negocio, nada resultado mas ilusorio, toda vez que la innovación es simplemente la capacidad intrínseca de toda empresa para descifrar en cada momento las demandas del mercado y evolucionar en función de sus grandes tendencias.

\section{Referencias Bibliográficas}

ARBELÁEZ-CAMPILLO, Diego Felipe; ANDREYEVNA DUDAREVA, Marianna; ROJAS-BAHAMÓN, Magda Julissa. 2019. "Las pandemias como factor perturbador del orden geopolítico en el mundo globalizado" En: Cuestiones Politicas. Disponible en línea. En: https:// produccioncientificaluz.org/index.php/cuestiones/article/view/31528. Fecha de consulta: 16/04/2020.

ARIAS, Fidias. G. 2006. El proyecto de investigación Introducción a la metodología científica. Editorial Episteme. Caracas, Venezuela.

BARRIOS, Karelis del Carmen; CONTRERAS SALINAS, Jheison A; OLIVEROVEGA, Enohemit. 2019. "La Gestión por Procesos en las Pymes de Barranquilla: Factor Diferenciador de la Competitividad Organizacional" En: Información Tecnológica. Disponible en linea. En: http://dx.doi. org/10.4067/So718-07642019000200103. Fecha de consulta: $12 / 12 / 2019$.

BOTELLO PENALOZA, Héctor Alberto. 2016. "Las certificaciones de calidad y la internacionalización de las firmas industriales colombianas" En: Suma de Negocios. Vol. 04, No. 16, pp. 73-81.

CADENA ECHEVERRIA, Jaime; AGUILAR, Mercedes; BUITRÓN, Pedro Enrique. 2018. "Competitividad en las pequeñas y medianas empresas del distrito Metropolitano de Quito" En: Small Business International Review. Vol. 02, No. 02, pp. 71-94. 
Marcia Jaramillo Paredes, Oscar Mauricio Romero Hidalgo, Martha Cecilia Aguirre Benalcázar y Jonathan Alexander Ruiz Carrillo

482

Influencia de la innovación en la competitividad de las medianas empresas del Ecuador

CARVACHE-FRANCO, Orly; GUTIÉRREZ CANDELA, Glenda; FRÍAS CASCO, Camilo. 2018. "Incidencia de la innovación y la tecnología en el desarrollo competitivo de las pequeñas y medianas empresas (PYMES) exportadoras de Guayas-Ecuador" En: Revista espacios. Vol. 39, No. 47, pp. 02-37.

CEPEDA PALACIO, Samuel David; VELÁSQUEZ ESTRADA, Libardo José. 2017. "Análisis evaluativo a los procesos de marketing en la internacionalización de las pequeñas y medianas empresas de alimentos de Medellín” En: Estudios Gerenciales. Vol. 33, No. 144, pp. 271-280.

DIDRIKSSON, Axel. 2015. "Política del conocimiento: contrapuntos" En: Economía Informa, Vol. 394, pp. 38-67.

DURÁN QUIRÓS, Alfredo; MORA ACEDO, Dennis; GONZÁLEZ LUTZ, María I; VARGAS HERNÁNDEZ, Guillermo. 2018. "Nivel de competitividad técnica de las empresas exportadoras de productos agrícolas no tradicionales de Costa Rica” En: Agronomía Costarricense. Vol. 24, No. 02, pp. 141-158.

FUENTES, Noe Aron; OSORIO, German; MUNGARAY, Alejandro. 2016. "Capacidades intangibles para la competitividad microempresarial en México” En: Revista UNAN. Vol. 47, No. 186, pp. 114-129.

GARCÍA GUILIANY, Jesús E; DURAN, Sonia E; CARDEÑO, Pórtela; PRIETO PULIDO, Ronald; GARCÍA CALI, Ernesto; PAZ Marcano, Annherys. 2017. "Proceso de planificación estratégica: Etapas ejecutadas en pequeñas y medianas empresas para optimizar la competitividad" En: Revista espacios. Vol. 38, No. 52, p. 16.

GARCÍA OCHOA, Juna José; LEÓN LARA, Juan de Dios; NUÑO DE LA PARRA, José Pablo. 2017. "Propuesta de un modelo de medición de la competitividad mediante análisis factorial" En: Contaduría y Administración. Vol. 62, No. 03, pp. 775-791.

GIL GAYTÁN, Olga Leticia. 2017. "La logística: clave para la competitividad global de las pequeñas y medianas empresas del estado de Jalisco en México” En: PAAKAT: Revista de Tecnologia y Sociedad. Vol. 06, No. 11.

GUTIÉRREZ RODRÍGUEZ, Ramon Eduardo; ALMANZA JUNCO, Carlos Alberto. 2016. "Una aproximación a la caracterización competitiva de los sectores productivos industrial y floricultor del municipio de Madrid Cundinamarca, Colombia” En: Suma de Negocios. Vol. 07, No. 16, pp. 82-93.

HORTA, Roberto; SILVEIRA, Luis; CAMACHO, Micaela. 2015. "Competitividad e innovación en la industria manufacturera en el Uruguay” En: Revista 
Iberoamericana de ciencia tecnológica y sociedad. Vol. 10, No. 28, pp. 23-49.

IBARRA CISNEROS, Manuel Alejandro; GONZALES TORRES, Lourdes Alicia; DEMUNER FLORES, María del Rosario. 2017. "Competitividad empresarial de las pequeñas y medianas empresas manufactureras de Baja California” En: Estudios Fronterizos. Vol. 18, No. 35, pp. 107-130.

INSTITUTO NACIONAL DE ESTADÍSTICAS Y CENSOS (INEC). 2016. Ecuador en cifras. Obtenido de Empresas en Ecuador: Encuesta estructural empresarial 2016. Disponible en línea. Es: https:// www.ecuadorencifras.gob.ec/documentos/web-inec/Estadisticas Economicas/Encuesta_Estructural_Empresarial/2016/Tomo_I/2016_ ENESEM_Metodologia.pdf. Fecha de consulta: 13/03/2019.

LEYVA CABRERA, Alma Brenda; CAVAZOS ARROYO, Judith; ESPEJEL BLANCO, Joel Enrique. 2018. "Influencia de la planeación estratégica y habilidades gerenciales como factores internos de la competitividad empresarial de las Pymes” En: Contaduría y administración. Vol. 63, No. 03, pp. 1-21.

LÓPEZ, D C. 2016. "Factores de calidad que afectan la productividad y competitividad de las micros, pequeñas y medianas empresas del sector industrial metalmecánico" En: Entre Ciencia e Ingeniería. Año 10, No. 20, pp. 99-107.

MOLINA YCAZA, Daniel Eduardo; SÁNCHEZ-RIOFRIO, Angélica María. 2016. "Factores de competitividad orientados a la pequeña y mediana empresa (PYME) en Latinoamérica: revisión de la literatura" En: Revista San Gregorio. No. 15, pp. 104-111.

MORA-RIAPIRA, Edwin; VERA-COLINA, Mary A; MELGAREJO-MOLINA, Zuray A. 2015. "Planificación estratégica y niveles de competitividad de las Mipymes del sector comercio en Bogotá” En: Estudios Gerenciales. Vol. 31, No. 134, pp. 79-87.

OSPINA ZAPATA, Melissa; PUCHE NIEVES, María Alejandra; ARANGO ÁLZATE, Bibiana. 2014. "Gestión de la Innovación en Pequeñas y Medianas Empresas. Generandoventajas competitivasy posicionamiento en el Mercado" En: Revista Electrónica Gestión de las Personas y Tecnología. Vol. 07, No. 19, pp. 56-67.

OTERO FLORES, Sergio; TADDEI BRINGAS, Cristina. 2018. "Competitividad de empresas familiares” En: Interciencia: Revista de ciencia y tecnología de América. Vol. 34, No. 04, pp. 236-241.

RUBIO, G; BUITRAGO, A. 2019. "Análisis de competitividad del sector 
Marcia Jaramillo Paredes, Oscar Mauricio Romero Hidalgo, Martha Cecilia Aguirre Benalcázar y Jonathan Alexander Ruiz Carrillo

484 Influencia de la innovación en la competitividad de las medianas empresas del Ecuador

microempresarial colombiano: una mirada con enfoque asociativo" En: REMIPE-Revista de Micro y Pequeñas Empresas e Empreendedorismo da Fatec Osasco. Vol. 05, No. 01, pp. 178 - 188.

SECRETARIA DE EDUCACIÓN SUPERIOR, CIENCIA, TECNOLOGÍA E INNOVACIÓN SENESCYT. 2019. Acuerdo para la innovación, clave del desarrollo económico de Ecuador. Disponible en line. En: https:// www.educacionsuperior.gob.ec/acuerdo-para-la-innovacion-clave-deldesarrollo-economico-de-ecuador/. Fecha de consulta: 12/02/2020.

TARAPUEZ, Edwin; GUZMÁN, Beatriz Elena; PARRA HERNÁNDEZ, Ramiro. 2019. "Estrategia e innovación en las Mipymes colombianas ganadoras del premio Innova 2010-2013” En: Estudios Gerenciales. Vol. 32, No. 139, pp. 170-180.

TOBAR-PESÁNTEZ, Luis. 2015. "Análisis competitivo de las pequeñas y medianas empresas en Cuenca Ecuador" En: Revista Internacional Administración \& Finanzas. Vol. 8, No. 03, pp. 79-92.

VÁZQUEZ ÁVILA, Guillermo; GUERRERO HERRERA, Juan Fernando; Núñez Moreno, Tania Emma. 2014. "Gestión de conocimiento, capital intelectual y competitividad en pymes manufactureras en México" En: Retos Revista de Ciencias de la Administración y Economía. Vol. 06, No. 07, pp. 28-43.

VILLASMIL ESPINOZA, Jorge Jesús. 2020. "La fragilidad de las civilizaciones humanas" En: Cuestiones Políticas. Disponible en línea. En: https://doi. org/10.46398/cuestpol.3764.00. Fecha de consulta: 20/06/2020. 

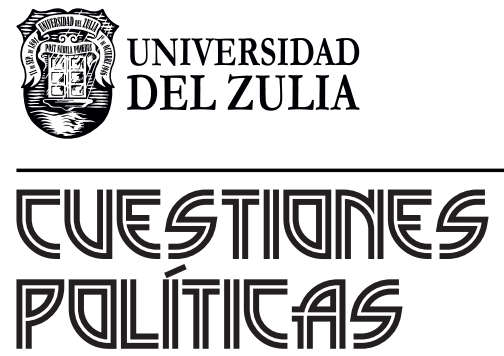

Vol.38 NEspecial

Esta revista fue editada en formato digital y publicada en diciembre de 2020, por el Fondo Editorial Serbiluz, Universidad del Zulia. Maracaibo-Venezuela 